\title{
BMJ Open Biomarkers for length of hospital stay, changes in muscle mass, strength and physical function in older medical patients: protocol for the Copenhagen PROTECT study - a prospective cohort study
}

Rikke S Kamper (D) ,1,2 Martin Schultz, ${ }^{3,2}$ Sofie K Hansen, ${ }^{1,2}$ Helle Andersen,,${ }^{4,2}$ Anette Ekmann, ${ }^{1,2}$ Hanne Nygaard, ${ }^{5,2}$ Fredrik Helland, ${ }^{6}$ Miriam R Wejse, ${ }^{6}$ Camilla B Rahbek, ${ }^{1}$ Tim Noerst, ${ }^{1}$ Eckart Pressel, ${ }^{6}$ Finn Erland Nielsen, ${ }^{5,7}$ Charlotte Suetta ${ }^{1,3}$

To cite: Kamper RS, Schultz M, Hansen SK, et al. Biomarkers for length of hospital stay, changes in muscle mass, strength and physical function in older medical patients: protocol for the Copenhagen PROTECT study - a prospective cohort study. BMJ Open 2020;10:e042786. doi:10.1136/ bmjopen-2020-042786

- Prepublication history for this paper is available online. To view these files, please visit the journal online (http://dx.doi. org/10.1136/bmjopen-2020042786).

Received 14 July 2020 Revised 08 December 2020 Accepted 11 December 2020

D) Check for updates

(c) Author(s) (or their employer(s)) 2020. Re-use permitted under CC BY-NC. No commercial re-use. See rights and permissions. Published by BMJ.

For numbered affiliations see end of article.

Correspondence to Rikke S Kamper; rikke.stefan.kamper.01@ regionh.dk

\section{ABSTRACT}

Introduction Sarcopenia is generally used to describe the age-related loss of muscle mass and strength believed to play a major role in the pathogenesis of physical frailty and functional impairment that may occur with old age. The knowledge surrounding the prevalence and determinants of sarcopenia in older medical patients is scarce, and it is unknown whether specific biomarkers can predict physical deconditioning during hospitalisation. We hypothesise that a combination of clinical, functional and circulating biomarkers can serve as a risk stratification tool and can (i) identify older acutely ill medical patients at risk of prolonged hospital stays and (ii) predict changes in muscle mass, muscle strength and function during hospitalisation. Method and analysis The Copenhagen PROTECT study is a prospective cohort study consisting of acutely ill older medical patients admitted to the acute medical ward at Copenhagen University Hospital, Bispebjerg and Frederiksberg, Denmark. Assessments are performed within 24 hours of admission and include blood samples, body composition, muscle strength, physical function and questionnaires. A subgroup of patients transferred to the Geriatric Department are included in a smaller geriatric cohort and have additional assessments at discharge to evaluate the relative change in circulating biomarker concentrations, body composition, muscle strength and physical function during hospitalisation. Enrolment commenced 4 November 2019, and proceeds until August 2021.

Ethics and dissemination The study protocol has been approved by the local ethics committee of Copenhagen and Frederiksberg $(\mathrm{H}-19039214)$ and the Danish Data Protection Agency (P-2019-239) and all experimental procedures were performed in accordance with the Declaration of Helsinki. Findings from the project, regardless of the outcome, will be published in relevant peer-reviewed scientific journals in online (www. clinicaltrials.gov).

Trial registration number NCT04151108
Strengths and limitations of this study

- A strength of the study is the large heterogeneous population, which brings generalisability to the study results.

- The assessments of physical function applied in the study have previously been evaluated in acutely admitted older medical patients.

- Bioelectrical impedance analysis may be affected by the hydration status of the patients.

- There are no direct measurements of the physical activity levels of the patients during admission.

- The study estimates stature by knee-height measurements, as many patients are unable to stand for height measurements.

\section{INTRODUCTION}

It is well-established that human skeletal muscle function declines with ageing, and sarcopenia is generally used to describe the age-related skeletal muscle atrophy and loss of muscle strength believed to play a major role in the pathogenesis of physical frailty, loss of independence and functional impairment that may occur with old age. ${ }^{1-3}$ Clinical sarcopenia has been defined in statistical terms assuming a lower normal limit of two SD below a mean relative appendicular muscle mass in young healthy adults. ${ }^{4}$ The prevalence of sarcopenia is estimated at 5\%-13\% in $60-70$ years old and $11 \%-50 \%$ in individuals aged 80 years or older. ${ }^{5}$ The aetiology of sarcopenia is complex and involves neuronal, hormonal, immunological and nutritional mechanisms. ${ }^{6-10}$ Furthermore, physical inactivity, chronic diseases, immobilisation and 
hospitalisation are known to play a part in the development of sarcopenia. ${ }^{611-13}$

In 2018, approximately $45 \%$ of all hospital admissions in Denmark concerned patients aged 65 or older who had a mean length of stay (LOS) of 3.5 days. ${ }^{14}$ Older patients are often inactive during hospitalisation spending $71 \%-83 \%$ of their time lying down, ${ }^{15} 16$ and at least $35 \%$ of older patients lose independence in one basic activity of daily living as an unintended consequence of a medical illness and hospitalisation. ${ }^{17}$ Sarcopenia may aggravate this functional decline, as patients with sarcopenia have an attenuated recovery of their functional levels 3 months following discharge ${ }^{18}$ From a clinical perspective, sarcopenia is associated with infectious complications, readmissions, increased need for rehabilitation following discharge, reduced quality of life, increased mortality and longer hospitalisation. ${ }^{419}$

Early mobilisation protocols have proven effective in reducing hospital-acquired disability and hospital length of stay. However, frequently reported barriers for implementation of early mobilisation include lack of staff and time to enable mobilisation of the patient. ${ }^{20}$ With an increasing ageing population and the heterogeneousness of older individuals, the systematic identification of older individuals at risk of prolonged hospitalisation and deconditioning during hospitalisation are of outmost importance. As such, the combination of clinical, functional and circulating biomarkers may serve as risk stratification tools to identify older patients at risk of these adverse outcomes.

\section{STUDY OBJECTIVES AND HYPOTHESES}

\section{Primary objectives and hypothesis}

We aim to examine whether circulating biomarkers at admission are associated with length of hospital stay in older ( $\geq 65$ years) acutely admitted medical patients and whether the combination of clinical and functional measures with these biomarkers can identify patients at risk of having a prolonged hospital stay ( $>96$ hours). In addition, we aim to establish circulating biomarkers associated with changes in muscle mass, muscle strength and function in geriatric patients during hospitalisation. We hypothesise that a combination of clinical and functional measures with circulating biomarkers has the potential to identify older ( $\geq 65$ years) acutely admitted medical patients at risk of prolonged ( $\geq 96$ hours) hospital stays and physical deconditioning during hospitalisation.

\section{Secondary objectives and hypothesis}

The secondary objectives are to determine whether circulating biomarkers are associated with readmissions within 90 days of discharge, frailty, discharge to a higher level of care and all-cause mortality within 90 days of the index admission and whether the combination of clinical and functional measures with these biomarkers can identify patients at risk of readmissions, discharge to a higher level of care and all-cause mortality. We hypothesise that a combination of clinical and functional measures with circulating biomarkers has the potential to identify older ( $\geq 65$ years) acutely admitted medical patients at risk of non-elective readmissions within 90 days of discharge, discharge to a higher level of care, and all-cause mortality within 90 days of the index admission.

\section{METHODS AND ANALYSIS}

\section{Setting and intervention}

The Copenhagen PROTECT study is a prospective cohort study consisting of acutely ill older medical patients admitted to the acute medical ward at Copenhagen University Hospital, Bispebjerg and Frederiksberg, Denmark. A subgroup of these patients, subsequently transferred to the Geriatric Department, are also included in a smaller geriatric cohort. Enrolment commenced 4 November 2019 and will proceed until August 2021.

\section{Eligible patients}

The current study is recruiting participants during a 1.5 -year period to avoid any seasonal differences in the patient population and to take into account the temporary pause in recruitment due to the COVID-19 pandemic. We aim to include a total of 1700 patients representing the PROTECT cohort, of which approximately 400 patients subsequently will be transferred to the Geriatric Department and constitute the Geriatric cohort. All patients admitted at the acute medical ward at Copenhagen University Hospital, Bispebjerg and Frederiksberg who fulfil the inclusion criteria and do not meet any exclusion criteria are eligible for the study (box 1). The hospital admission during which the patient is recruited represents the index admission. Any subsequent non-elective admissions of included patients during the study inclusion period will be interpreted as readmissions. Included patients will be followed for 90 days following discharge from index admission to investigate future readmissions and mortality.

\section{Outcomes}

The primary outcome in the PROTECT cohort is the length of hospital stay. Successive events of hospitalisation have been suggested to contribute to the development of sarcopenia, and even short periods (4-5 days) of skeletal

\section{Box 1 Inclusion and exclusion criteria}

Inclusion criteria

Equal to or over the age of 65 years.

Acutely admitted with a medical diagnosis (ie, non-surgical).

Exclusion criteria

Admitted for more than 24 hours prior to baseline assessment.

Terminal illness (expected life span of less than 6 months)

Temporary civil registration number.

Droplet or airborne infections requiring isolation.

Does not speak or read Danish.

Patients judged medically contraindicated by health personnel. Inability to provide informed consent for participation. 
Box 2 Primary and secondary outcomes in the PROTECT cohort
Primary outcome
Length of hospital stay.
Secondary outcomes
Non-elective readmissions within 90 days of discharge.
All-cause mortality within 90 days of index admission.
In-hospital mortality.
Muscle mass at admission.
Muscle strength at admission.
Muscle function at admission.
Frailty.

muscle disuse are known to induce muscle atrophy. ${ }^{21} 22$ In 2018, the mean length of hospital stay in Denmark was 84 hours in patients aged 65 years or over. ${ }^{14}$ while the mean LOS in the two largest local hospitals was 96 hours. As such, we have defined a prolonged hospital length of stay as an admission lasting $>96$ hours.

The primary outcomes in the Geriatric cohort are the relative changes in muscle mass, muscle strength and muscle function during hospitalisation. Primary and secondary outcomes for the PROTECT cohort and the Geriatric cohort are listed in boxes 2 and 3, respectively.

We have defined geriatric patients discharged to an increased level of care as (i) patients receiving increased relief in terms of walking aids or patients with an increased need for caregiver assistance or home care, (ii) patients referred to rehabilitation or 24 hours care or (iii) patients moving to a nursing home following discharge. Data on readmissions will be limited to non-elective readmissions in Region Zealand and the Capital Region of Denmark. A geriatrician will evaluate whether the readmission is related to the index admission; that is, newly emerged acute illness following the index admission, acute aggravation of disease treated during the index admission or complication to treatment during the index admission.

\section{Assessment and randomisation}

The research personnel might be unable to assess all patients, as the number of eligible patients (ie, fulfilling inclusion criteria with the absence of exclusion criteria)

\section{Box 3 Primary and secondary outcomes in the Geriatric} cohort

\section{Primary outcomes}

Changes in muscle mass during hospitalisation.

Changes in muscle strength during hospitalisation.

Changes in muscle function during hospitalisation.

Secondary outcomes

Length of hospital stay.

Non-elective readmissions within 90 days of discharge.

All-cause mortality within 90 days of index admission.

In-hospital mortality.

Discharge to an increased level of care.

Frailty. varies daily. Thus, to avoid selection bias, all eligible patients on the day in question are randomised using a computer-generated randomisation sequence to establish a randomised visitation sequence. Patients who wish to participate sign an informed consent and baseline measurements are performed within the first 24 hours of admission. All included patients have blood samples drawn to determine concentrations of tumor necrosis factor (TNF)- $\alpha$, interleukin (IL)-6, IL-10, transforming growth factor (TGF)- $\beta 1$, follistatin, insulin-like growth factor (IGF)-1, growth differentiation factor (GDF)-11, GDF-15 and soluble urokinase-type plasminogen activator receptor (suPAR).

Handgrip strength is assessed using a digital hand-held dynamometer (Model SH1001; SAEHAN Corporation, Yangdeok-Dong, Masan, South Korea). Patients able to leave the bed sit on a chair with the elbow flexed at $90^{\circ}$ and the wrist in a neutral position, while bedridden patients are assessed in the hospital bed with the backrest elevated. The highest value of three attempts with the dominant hand is used for analyses. Should the third trial elicit the highest value, the patient continues until a lower value is achieved. Muscle function is assessed in the $30 \mathrm{~s}$ sit-to-stand test, where patients are asked to stand up from a standardised chair as many times as possible with their arms folded across the chest. Only full standing positions are counted. ${ }^{23}{ }^{24}$ Patients included in the Geriatric cohort also have their habitual gait-speed assessed. The gait-speed assessment is measured over a course of $4 \mathrm{~m}$ and includes walking aids if they are used by the patient. Patients stand behind a starting line and are asked to start walking towards a visual goal at their habitual pace. The visual goal is placed after $5.5 \mathrm{~m}$. to reduce the effect of deceleration. The fastest of the two attempts will be used for analyses and quantified as $\mathrm{m} / \mathrm{s}{ }^{23}$ The assessment of handgrip strength $(\mathrm{kg})$ and habitual gait-speed have previously shown to be feasible and reliable measures in acutely older medical patients. However, the feasibility and reliability of the $30 \mathrm{~s}$ sit-to-stand test was moderate, as only half of the patients were able to perform the test as instructed. ${ }^{23}$ Thus, we have included an additional nominal variable to categorise the sit-to-stand ability as either (i) able to perform the test as instructed, (ii) ability to rise using the armrest and (iii) inability to rise independently from a chair.

Bodyweight $(\mathrm{kg})$ is assessed using chair scales and height $(\mathrm{cm})$ is estimated with a segmometer using the kneeheight measurement and age with the equations from Chumlea $e t a l{ }^{25}$ Body composition, including whole body phase angle, is assessed using Direct-Segmental Multifrequency Bioelectrical Impedance Analyses (DSM-BIA) (InBody S10; Biospace, Seoul, Korea), which has previously been used in elderly acutely admitted patients with a mean LOS of 5 days. ${ }^{26}$ Self-reported current smoking is reported as a dichotomous variable. Patients included in the Geriatric cohort are also assessed at discharge to evaluate circulating biomarker concentrations as well as changes in body composition, muscle strength and 
functional performance. Tests of strength, physical function and body composition measurements are performed by trained research personnel. The presence of frailty is assessed by trained nurses associated with the study using the Canadian Study of Health and Aging Clinical Frailty Scale. ${ }^{27}$ Patients are screened for sarcopenia using the SARC-F questionnaire, ${ }^{28}$ while cognitive status is evaluated by the short Orientation-Memory-Concentration test. ${ }^{29}$ The risk of malnutrition is assessed and validated using the Short Nutritional Assessment Questionnaire. ${ }^{30}$ A flowchart showing the timeline and assessments in the two cohorts can be seen in figure 1 .

Information on medical treatment is evaluated by counting all prescribed medications, including unscheduled medications, except for the following:

- Eyedrops.

- Eardrops.

- Lotions and ointments.

- Antibiotic treatment of limited duration.

- Multivitamins.

- Supplementary nutrition or tube feeding.

Medications listed two times containing the same substance are only counted once. Comorbidity is evaluated by the Charlson Comorbidity Index ${ }^{31}$ and obtained by evaluating the type and number of International Classification of Diseases (ICD)-10 discharge diagnosis during the last 5 years of the index admission. Sepsis is defined in accordance with the Sepsis-3 criteria. ${ }^{32}$ Data on emigration and all-cause mortality within 90 days of index admission is extracted from the Danish Civil Registration System. A summary of variables assessed by research personnel and extracted from the electronic patient system (EPIC) or the Danish Civil Registration System are listed in table 1.

\section{Data management}

Following data acquisition, all physical documents are stored in accordance with the guidelines for data management from the Danish Data Protection Agency. Electronic data are managed and stored using Research Electronic Data Capture (REDCap), ${ }^{33} 34$ a web-based secure software platform hosted at Bispebjerg-Frederiksberg University Hospital. To ensure data quality, the REDCap database was built to ensure data integrity including real-time data validation, integrity checks and range checks for data values.

\section{Patient and public involvement}

On request, patients with measures of muscle mass, strength or function can gain insight into their values and receive advice to improve from either an exercise physiologist or a physiotherapist. Patients are not involved in the study design, recruitment or other aspects of the study.

\section{Power calculation and statistics}

To evaluate the prognostic abilities of circulating biomarkers (individually, in combination and combined with clinical and functional measures) we will use the area under the curve for receiver operating characteristics
(AUROC) statistics. A reference group of 2058 patients over the age of 65 from Bispebjerg-Frederiksberg University Hospital and Herlev-Gentofte Hospital had a mean age of 78.3 years and a mean length of stay of 5.8 days during hospitalisation. In these patients, 817 (39.7\%) had a prolonged length of stay, defined as a hospitalisation lasting more than 96 hours. With a sample size of 1700 and the assumption that approximately $40 \%$ of older medical patients have a prolonged hospital stay, an AUROC of 82 will have a power of 0.9 with a significance level of 0.05 .

A table of summary statistics will be presented with baseline variables. Continuous variables will be summarised with the following: $\mathrm{n}$ (non-missing sample size), mean, $\mathrm{SD}$, median, IQR and number of missing values. Categorical variables will be reported as frequency and percentages (based on non-missing sample size), and number of missing values. Data missing at random will be imputed using multiple imputation.

To evaluate whether clinical, functional and circulating biomarkers are associated with length of stay we will perform multivariate logistic regression. Patients will be grouped in either normal ( $<96$ hours) or extended length of stay ( $\geq 96$ hours) and Cox regression analysis will be used to compare differences in non-elective readmission and all-cause mortality. Patients will be followed from the date of discharge from the index admission until the end of the follow-up period, emigration, readmission, or death as appropriate. To assess the discriminative ability of biomarkers with regards to an extended length of stay and all-cause mortality, we will use the area under the curve (AUC) for receiver operating characteristics (ROC) curves. AUCs for different ROC curves will be compared using the DeLong test. The association of circulating biomarkers with changes in muscle mass, muscle strength and function in the geriatric cohort will be assessed using a multivariate linear model adjusted for the relative length of stay.

\section{Study organisation}

The study is a researcher initiated clinical study. The protocol was written by the steering committee composed of experts in geriatric medicine and acute medicine and a $\mathrm{PhD}$ student in basic and clinical research in musculoskeletal sciences. The committee is responsible for the design of the study, supervision of research personnel, data acquisition, communication and publication of results, approval of substudies and ensuring that future studies comply with the regulations regarding data management.

At present (July 2020), the study has included 377 patients, of which 62 are part of the geriatric cohort. Inclusion was temporarily paused due to the impact of the COVID-19 pandemic.

\section{ETHICS AND DISSEMINATION}

All procedures are being conducted according to 'Good Clinical Practice' standards, regarding initiation, monitoring and reporting. The study protocol has been 


\section{Acutely admitted medical patients ( $\geq 65$ years)}

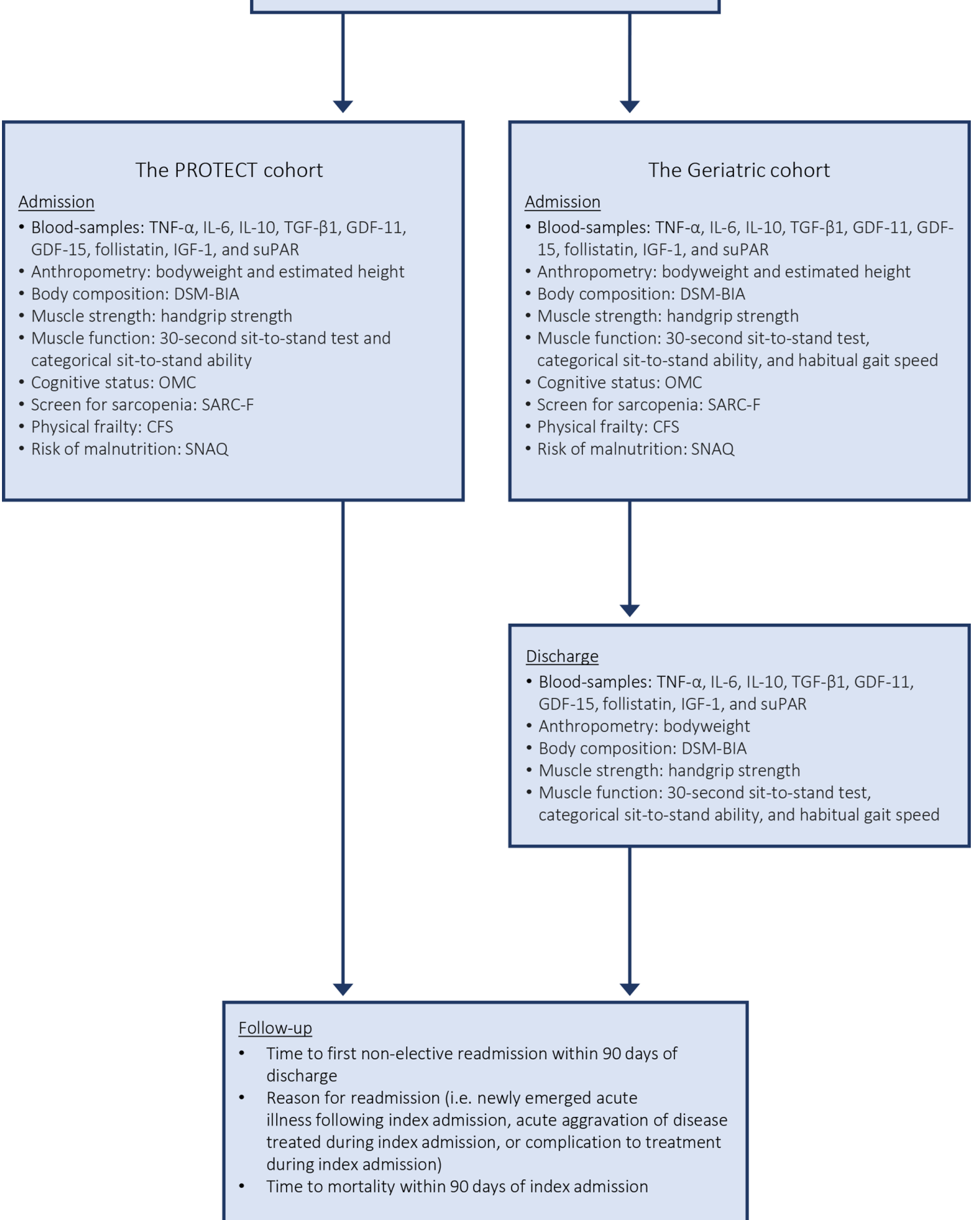

Figure 1 Timeline and assessments in the PROTECT cohort and the Geriatric cohort. CFS, Clinical Frailty Scale; DSMBIA, Direct-Segmental Multi-frequency Bioelectrical Impedance Analyses; GDF, growth differentiation factor; IGF, insulin-like growthfactor; IL, interleukin; OMC, Orientation-Memory-Concentration test; TNF, tumor necrosis factor; SARC-F, sarcopenia screening; SNAQ, Short Nutritional Assessment Questionnaire.

approved by the local ethics committee of Copenhagen and Frederiksberg (H-19039214) and the Danish Data Protection Agency (P-2019-239) and all experimental procedures are performed in accordance with the Declaration of Helsinki. The project complies with the regulations of the General Data Protection Regulation and the Data Protection Act.

All eligible patients receive oral and written information. In the case of severe dementia or delirium, some patients might be unable to provide participant consent. 
Table 1 Variables assessed by research personnel and extracted from EPIC or the Danish civil registration system

Extracted from EPIC or the Danish Civil Assessed by research personnel Registration System

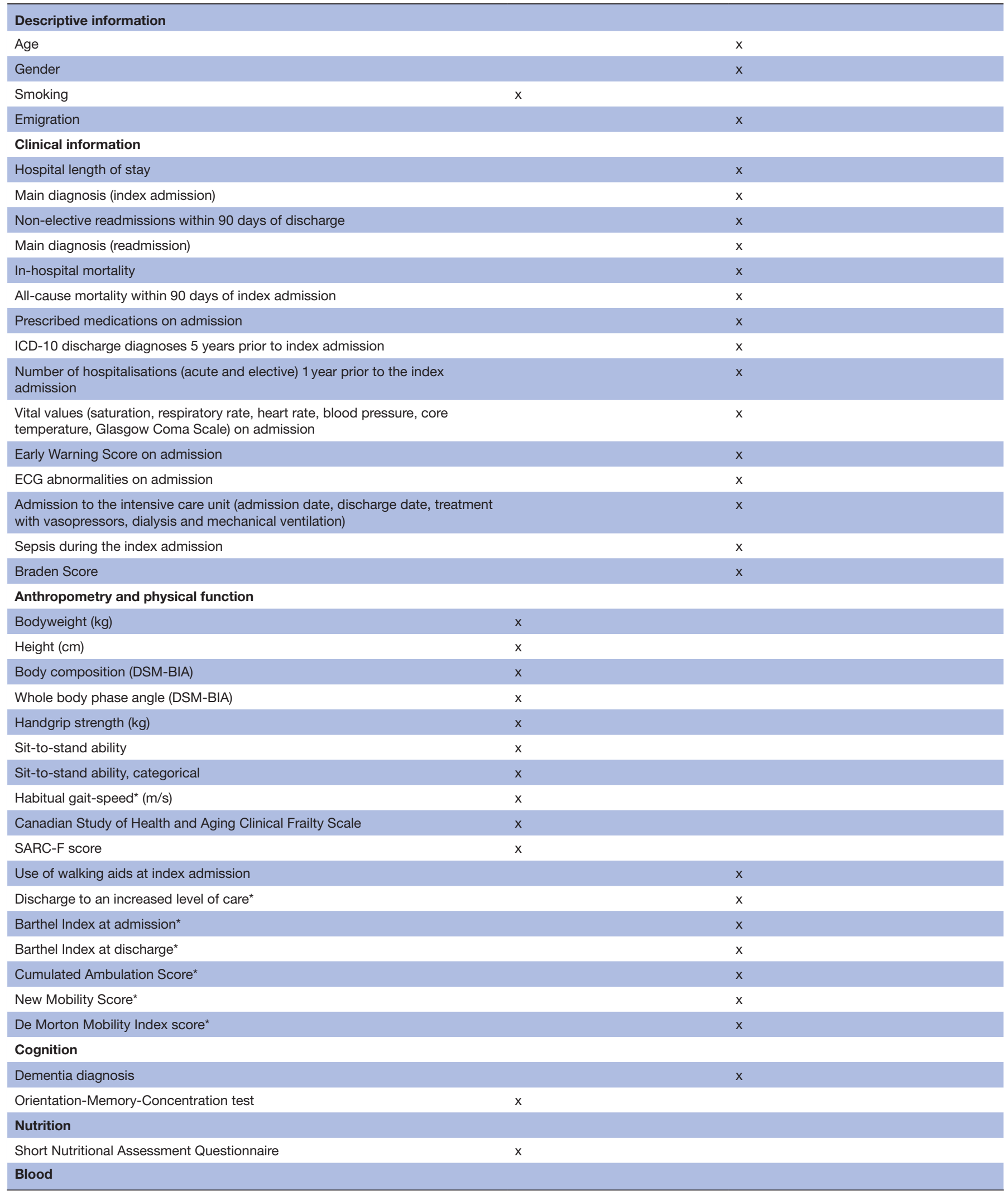




\section{Results of PROTECT blood tests (TNF- $\alpha$, IL-6, IL-10, TGF- $\beta$, GDF-11, GDF-15,}

follistatin, IGF-1 and suPAR)

*Variables included in the Geriatric cohort only.

DSM-BIA, Direct-Segmental Multi-frequency Bioelectrical Impedance Analyses; GDF, growth differentiation factor; ICD, International Classification of Diseases; IGF, insulin-like growth factor; IL, interleukin; SARC-F, sarcopenia screening; TGF, transforming growth factor; TNF, tumor necrosis factor.

In these cases, we seek participant consent from a close relative or guardian, should the guardianship include access to sign participant consent for research purposes. Independent medical doctors who have knowledge of the project but are not associated with the project and are independent of the interests of the principal investigator evaluate whether these subjects can participate in the study.

The project entails minimal discomfort and no permanent side effects; thus, it is considered ethically sound. Findings from the project, regardless of the outcome, will be published in relevant peer-reviewed scientific journals ensuring the anonymity of the patients. The study is registered at clinicaltrials.gov where positive, negative or inconclusive results will be published.

\section{DISCUSSION}

The importance of sarcopenia has recently been underlined by its inclusion as a reportable disease in the Centers for Disease Control and Prevention (ICD-10-CM code M62.84) in October 2016. ${ }^{35}$ Even though the serious consequences of sarcopenia are widely recognised, the diagnose has yet to be implemented in clinical practice in Denmark. Lack of knowledge regarding the basic biological mechanisms driving sarcopenia in conjunction with other age-related diseases and lack of systematic assessment hinders the identification and treatment of sarcopenia and can lead to physical deconditioning during hospitalisation. As such, the knowledge surrounding the prevalence and determinants of sarcopenia in older medical patients is scarce, and it is unknown whether circulating biomarkers, individually or in combination, can predict physical deconditioning during hospitalisation.

Mechanisms that regulate skeletal muscle mass are central to the understanding of sarcopenia. Myostatin, also referred to as GDF- 8 , is a part of the TGF- $\beta$ family and predominantly expressed in skeletal muscle. Myostatin and TGF- $\beta$ are inducers of catabolic processes, inhibiting muscle growth and inducing muscle protein breakdown via activation of the Small Mothers Against Decapentaplegic (SMAD)2 and SMAD3 transcription factors. ${ }^{3637}$ Myostatin is reportedly increased with ageing, ${ }^{38}$ and following prolonged bed rest, ${ }^{39}$ and treatment with myostatin antibodies attenuates the loss of muscle mass and function induced by immobilisation in mice. ${ }^{40}$

Recently, GDF-11, a TGF- $\beta$ family ligand, ${ }^{41}$ has been measured in human blood samples. ${ }^{42}$ High circulating GDF-11 levels have been related to increased disease burden and elevated risk of postoperative complications and mortality in older adults undergoing heart surgery. Notably, patients categorised as physically frail based on low handgrip strength and gait speed as well as self-reported activity measures had significantly higher GDF-11 levels compared with non-frail controls. ${ }^{42}$

GDF-15, another member of the TGF- $\beta$ family, is present in low levels under healthy conditions but can increase during disease or injury and contribute to muscle wasting by suppressing appetite, which may result in anorexia and drastic weight loss. ${ }^{43}$ In older patients, unintentional weight loss has been associated with an increased in-hospital morbidity and increased overall mortality. ${ }^{44}$ GDF-15 may be induced in response to cellular stress signals or dysfunctions, and it has been suggested that circulating levels of GDF-15 could be biomarker of mitochondrial dysfunction. ${ }^{45}$ Nonetheless, several studies demonstrate that GDF-15 levels are predictors of all-cause mortality. ${ }^{467}$

Follistatin acts as an antagonist to TGF- $\beta$ family ligands including myostatin, TGF- $\beta$ and GDF- $11 .{ }^{37}$ Measurements of TGF- $\beta$ ligands as well as their antagonist follistatin could represent biomarkers of muscle breakdown, physical function or mortality. However, the translation of these findings into clinical utility needs further validation in a larger cohort.

Several studies have investigated the association of inflammatory biomarkers with muscle mass, muscle strength and muscle function in healthy older subjects. Most commonly, studies have focused on a few biomarkers, such as TNF- $\alpha$, IL-6 or C-reactive protein (CRP). ${ }^{9}$ 48-50 A recent study has demonstrated an inverse relationship between a composite of proinflammatory and antiinflammatory markers and muscle mass, strength and function in healthy older subjects. ${ }^{51}$ However, results are inconsistent and lack clear evidence as to whether these inflammatory biomarkers are associated with sarcopenia. Nonetheless, circulating levels of CRP are predictive of both the length of hospital stay and readmissions. ${ }^{52} 53$ Indeed, geriatric patients with inflammation, evaluated by 
CRP levels at admission, stayed on average 3 days longer than patients without inflammation. ${ }^{54}$

The anabolic growth factor, IGF-1, and the IGF-1/phosphatidylinositol 3-kinase(PI3K)/Akt pathway is involved in skeletal muscle hypertrophy and atrophy. ${ }^{556} \mathrm{Circu}-$ lating IGF-1 levels decrease with ageing, while inflammatory markers such as TNF- $\alpha$ and IL- 6 can interfere with the IGF-1 signalling pathway. ${ }^{57}$ As such, changes in IGF-1/PI3K/Akt signalling during ageing may be a result of decreased IGF-1 expression as well as IGF-1 inhibition. Notably, no difference in circulating IGF-1 concentrations were found between older sarcopenic and non-sarcopenic women. ${ }^{59}$

Recently, suPAR was established as a biomarker of inflammation and immune activation, and elevated levels of suPAR are believed to reflect a state of chronic inflammation. ${ }^{60}$ SuPAR correlates with other inflammatory markers, such as TNF- $\alpha$, and patients with the highest levels of suPAR generally have the worst prognosis. ${ }^{61}$ In one study, suPAR was associated with low muscle mass, while IL-6 was associated with low muscle mass and increased fat mass in both patients and healthy controls. ${ }^{62}$ Thus, there seem to be distinct inflammatory processes occurring simultaneously with different effects on muscle mass and fat mass, respectively.

Distinct patient populations with coexisting pathophysiological processes might exhibit different biomarker profiles. Further validation needs to be conducted in different patient populations to use the possible prognostic value of these biomarkers, either individually, or in combination with functional and clinical measures. Systematic identification of patients at risk of prolonged hospitalisation and deconditioning should occur to enable early individualised interventions to counteract the adverse outcomes of prolonged bed rest.

Results from the Copenhagen PROTECT study can be helpful in the identification of older patients at risk of prolonged hospitalisation. Additionally, circulating biomarker assays able to predict physical deconditioning during hospitalisation will help in the early detection of geriatric patients at risk of deconditioning during hospitalisation. This knowledge can then be tested in a future interventional study. The Copenhagen PROTECT study is considered feasible, ethically sound, and with potential extensive implications for future identification and treatment of sarcopenia in older medical patients.

\section{Author affiliations}

${ }^{1}$ Geriatric Research Unit, Department of Geriatric and Palliative Medicine, Copenhagen University Hospital, Bispebjerg and Frederiksberg, Copenhagen, Denmark

${ }^{2}$ CopenAge; Copenhagen Center for Clinical Age Research, University of Copenhagen, Copenhagen, Denmark

${ }^{3}$ Geriatric Research Unit, Department of Medicine, Copenhagen University Hospital, Herlev and Gentofte, Herlev, Denmark

${ }^{4}$ Department of Occupational and Physiotherapy, Copenhagen University Hospital, Bispebjerg and Frederiksberg, Copenhagen, Denmark

${ }^{5}$ Department of Emergency Medicine, Copenhagen University Hospital, Bispebjerg and Frederiksberg, Copenhagen, Denmark
${ }^{6}$ Department of Geriatric and Palliative Medicine, Copenhagen University Hospital, Bispebjerg and Frederiksberg, Copenhagen, Denmark

${ }^{7}$ Copenhagen Center for Translational Research, Copenhagen University Hospital, Bispebjerg and Frederiksberg, Copenhagen, Denmark

Correction notice This article has been corrected since it first published. The provenance and peer review statement has been included.

Acknowledgements The authors would like to thank the doctors, nurses, secretaries and physiotherapists at the acute ward and the Department of Geriatric and Palliative Medicine for their cooperation and patience during patient recruitment and assessment. Furthermore, they would like to thank Rasmus Rønnemoes Iversen for his help in the recruitment of patients.

Contributors RSK, CS, MS and FEN drafted the manuscript. CS, FEN, RSK, MS, AE, $\mathrm{HN}, \mathrm{HA}, \mathrm{MRW}$ and EP designed the study. SKH, HA, HN, AE, FH, MRW, EP, TN and CBR contributed to the discussion, edited and reviewed the manuscript. All authors read and approved the final manuscript.

Funding The work is supported by funding from the Novo Nordisk Foundation; grant number NNF180C0052826.

Disclaimer The funders take no part in the design of the study; in the collection, analysis or interpretation of data; in the writing of manuscripts or decisions regarding publication.

\section{Competing interests None declared.}

Patient and public involvement Patients and/or the public were not involved in the design, or conduct, or reporting, or dissemination plans of this research.

Patient consent for publication Not required.

Provenance and peer review Not commissioned; externally peer reviewed.

Open access This is an open access article distributed in accordance with the Creative Commons Attribution Non Commercial (CC BY-NC 4.0) license, which permits others to distribute, remix, adapt, build upon this work non-commercially, and license their derivative works on different terms, provided the original work is properly cited, appropriate credit is given, any changes made indicated, and the use is non-commercial. See: http://creativecommons.org/licenses/by-nc/4.0/.

ORCID iD

Rikke S Kamper http://orcid.org/0000-0002-1927-9078

\section{REFERENCES}

1 Rosenberg IH. Sarcopenia: origins and clinical relevance. Clin Geriatr Med 2011;27:337-9.

2 Roubenoff R. Sarcopenia: a major modifiable cause of frailty in the elderly. J Nutr Health Aging 2000;4:140-2.

3 Rantanen T, Avlund K, Suominen H. Muscle strength as a predictor of onset of ADL dependence in people aged 75 years. Aging Clin Exp Res 2002;14:10-15.

4 Cruz-Jentoft AJ, Baeyens JP, Bauer JM, et al. Sarcopenia: European consensus on definition and diagnosis: report of the European Working group on sarcopenia in older people. Age Ageing 2010;39:412-23.

5 von Haehling S, Morley JE, Anker SD. An overview of sarcopenia: facts and numbers on prevalence and clinical impact. J Cachexia Sarcopenia Muscle 2010;1:129-33.

6 Roubenoff R, Heymsfield SB, Kehayias JJ, et al. Standardization of Nomenclature of body composition in weight loss. Am J Clin Nutr 1997;66:192-6.

7 Aagaard P, Suetta C, Caserotti P, et al. Role of the nervous system in sarcopenia and muscle atrophy with aging: strength training as a countermeasure. Scand J Med Sci Sports 2010;20:49-64.

8 La Colla A, Pronsato L, Milanesi L, et al. 17ß-Estradiol and testosterone in sarcopenia: Role of satellite cells. Ageing Res Rev 2015;24:166-77.

9 Bano G, Trevisan C, Carraro S, et al. Inflammation and sarcopenia: a systematic review and meta-analysis. Maturitas 2017;96:10-15.

10 Cruz-Jentoft AJ, Kiesswetter E, Drey M, et al. Nutrition, frailty, and sarcopenia. Aging Clin Exp Res 2017;29:43-8.

11 Bautmans I, Van Puyvelde K, Mets T. Sarcopenia and functional decline: pathophysiology, prevention and therapy. Acta Clin Belg 2009;64:303-16

12 Suetta C, Hvid LG, Justesen L, et al. Effects of aging on human skeletal muscle after immobilization and retraining. J Appl Physiol 2009;107:1172-80. 
13 Gill TM, Allore HG, Holford TR, et al. Hospitalization, restricted activity, and the development of disability among older persons. JAMA 2004;292:2115-24.

14 Denmark S. Available: https://www.statistikbanken.dk/IND05 [Accessed July 2020].

15 Brown CJ, Redden DT, Flood KL, et al. The underrecognized epidemic of low mobility during hospitalization of older adults. J Am Geriatr Soc 2009;57:1660-5.

16 Pedersen MM, Bodilsen AC, Petersen J, et al. Twenty-Four-Hour mobility during acute hospitalization in older medical patients. $J$ Gerontol A Biol Sci Med Sci 2013;68:331-7.

17 Covinsky KE, Palmer RM, Fortinsky RH, et al. Loss of independence in activities of daily living in older adults hospitalized with medical illnesses: increased vulnerability with age. J Am Geriatr Soc 2003:51:451-8

18 Sánchez-Rodríguez D, Marco E, Miralles R, et al. Sarcopenia, physical rehabilitation and functional outcomes of patients in a subacute geriatric care unit. Arch Gerontol Geriatr 2014;59:39-43.

19 Peterson SJ, Braunschweig CA. Prevalence of sarcopenia and associated outcomes in the clinical setting. Nutr Clin Pract 2016;31:40-8.

20 Surkan MJ, Gibson W. Interventions to mobilize elderly patients and reduce length of hospital stay. Can J Cardiol 2018;34:881-8.

21 Kouw IWK, Groen BBL, Smeets JSJ, et al. One week of hospitalization following elective hip surgery induces substantial muscle atrophy in older patients. J Am Med Dir Assoc 2019;20:35-42.

22 Suetta C, Frandsen U, Jensen L, et al. Aging affects the transcriptional regulation of human skeletal muscle disuse atrophy. PLoS One 2012;7:e51238.

23 Bodilsen AC, Juul-Larsen HG, Petersen J, et al. Feasibility and interrater reliability of physical performance measures in acutely admitted older medical patients. PLoS One 2015;10:e0118248.

24 Suetta C, Haddock B, Alcazar J, et al. The Copenhagen sarcopenia study: lean mass, strength, power, and physical function in a Danish cohort aged 20-93 years. J Cachexia Sarcopenia Muscle 2019:10:1316-29.

25 Chumlea WC, Roche AF, Steinbaugh ML. Estimating stature from knee height for persons 60 to 90 years of age. J Am Geriatr Soc 1985;33:116-20.

26 Van Ancum JM, Scheerman K, Pierik VD, et al. Muscle strength and muscle mass in older patients during hospitalization: the empower study. Gerontology 2017;63:507-14.

27 Rockwood K, Song X, MacKnight C, et al. A global clinical measure of fitness and frailty in elderly people. CMAJ 2005;173:489-95.

28 Malmstrom TK, Miller DK, Simonsick EM, et al. SARC-F: a symptom score to predict persons with sarcopenia at risk for poor functional outcomes. J Cachexia Sarcopenia Muscle 2016;7:28-36.

29 Katzman R, Brown T, Fuld P, et al. Validation of a short OrientationMemory-Concentration test of cognitive impairment. Am J Psychiatry 1983;140:734-9.

30 Kruizenga HM, Seidell JC, de Vet HCW, et al. Development and validation of a hospital screening tool for malnutrition: the short nutritional assessment questionnaire (SNAQ). Clin Nutr 2005;24:75-82.

31 Charlson ME, Pompei P, Ales KL, et al. A new method of classifying prognostic comorbidity in longitudinal studies: development and validation. J Chronic Dis 1987:40:373-83.

32 Singer M, Deutschman CS, Seymour CW, et al. The third International consensus definitions for sepsis and septic shock (Sepsis-3). JAMA 2016;315:801-10.

33 Harris PA, Taylor R, Thielke R, et al. Research electronic data capture (REDCap)--a metadata-driven methodology and workflow process for providing translational research informatics support. J Biomed Inform 2009;42:377-81.

34 Harris PA, Taylor R, Minor BL, et al. The REDCap Consortium: building an international community of software platform partners. $J$ Biomed Inform 2019;95:103208.

35 World Health Organization International statistical classification of diseases and related health problems. - 10th revision, Fifth edition, 2016. Available: https://icd.who.int/browse10/2016/en\#/M60-M63 [Accessed uly 2020].

36 Lee S-J, Reed LA, Davies MV, et al. Regulation of muscle growth by multiple ligands signaling through activin type II receptors. Proc Nat Acad Sci U S A 2005;102:18117-22.

37 Han HQ, Zhou X, Mitch WE, et al. Myostatin/activin pathway antagonism: molecular basis and therapeutic potential. Int J Biochem Cell Biol 2013;45:2333-47.
38 Yarasheski KE, Bhasin S, Sinha-Hikim I. Serum myostatinimmunoreactive protein is increased in 60-92 year old women and men with muscle wasting. J Nutr Health Aging 2002;6:343-8.

39 Reardon KA, Davis J, Kapsa RM, et al. Myostatin, insulin-like growth factor-1, and leukemia inhibitory factor mRNAs are upregulated in chronic human disuse muscle atrophy. Muscle Nerve 2001;24:893-9.

40 Murphy KT, Cobani V, Ryall JG, et al. Acute antibody-directed myostatin inhibition attenuates disuse muscle atrophy and weakness in mice. J Appl Physiol 2011;110:1065-72.

41 Nakashima M, Toyono T, Akamine A, et al. Expression of growth/ differentiation factor 11, a new member of the BMP/TGFbeta superfamily during mouse embryogenesis. Mech Dev 1999;80:185-9.

42 Schafer MJ, Atkinson EJ, Vanderboom PM, et al. Quantification of Gdf11 and myostatin in human aging and cardiovascular disease. Cell Metab 2016;23:1207-15.

43 Tsai VWW, Husaini Y, Manandhar R, et al. Anorexia/Cachexia of chronic diseases: a role for the TGF- $\beta$ family cytokine MIC-1/GDF15. $J$ Cachexia Sarcopenia Muscle 2012;3:239-43.

44 Gaddey HL, Holder K. Unintentional weight loss in older adults. Am Fam Physician 2014;89:718-22.

45 Fujita Y, Taniguchi Y, Shinkai S, et al. Secreted growth differentiation factor 15 as a potential biomarker for mitochondrial dysfunctions in aging and age-related disorders. Geriatr Gerontol Int 2016;16 Suppl 1:17-29.

46 Eggers KM, Kempf T, Wallentin L, et al. Change in growth differentiation factor 15 concentrations over time independently predicts mortality in community-dwelling elderly individuals. Clin Chem 2013;59:1091-8.

47 Wiklund FE, Bennet AM, Magnusson PKE, et al. Macrophage inhibitory cytokine-1 (MIC-1/GDF15): a new marker of all-cause mortality. Aging Cell 2010;9:1057-64.

48 Schaap LA, Pluijm SMF, Deeg DJH, et al. Inflammatory markers and loss of muscle mass (sarcopenia) and strength. Am J Med 2006;119:526.e9-526.e17.

49 Visser M, Pahor M, Taaffe DR, et al. Relationship of interleukin-6 and tumor necrosis factor-alpha with muscle mass and muscle strength in elderly men and women: the health ABC study. J Gerontol A Biol Sci Med Sci 2002;57:M326-32.

50 Cesari M, Penninx BWJH, Pahor M, et al. Inflammatory markers and physical performance in older persons: the InCHIANTI study. $J$ Gerontol A Biol Sci Med Sci 2004;59:M242-8.

51 Calvani R, Marini F, Cesari M, et al. Systemic inflammation, body composition, and physical performance in old community-dwellers. $J$ Cachexia Sarcopenia Muscle 2017;8:69-77.

52 Brown SH, Flint K, Storey A, et al. Routinely assessed biochemical markers tested on admission as predictors of adverse outcomes in hospitalized elderly patients. Hosp Pract 2012;40:193-201.

53 Hogarth MB, Gallimore R, Savage P, et al. Acute phase proteins, Creactive protein and serum amyloid a protein, as prognostic markers in the elderly inpatient. Age Ageing 1997;26:153-8.

54 Bautmans I, Njemini R, Lambert M, et al. Circulating acute phase mediators and skeletal muscle performance in hospitalized geriatric patients. J Gerontol A Biol Sci Med Sci 2005;60:361-7.

55 Bodine SC, Stitt TN, Gonzalez M, et al. Akt/mTOR pathway is a crucial regulator of skeletal muscle hypertrophy and can prevent muscle atrophy in vivo. Nat Cell Biol 2001;3:1014-9.

56 Glass DJ. Skeletal muscle hypertrophy and atrophy signaling pathways. Int J Biochem Cell Biol 2005;37:1974-84.

57 Barbieri M, Ferrucci L, Ragno E, et al. Chronic inflammation and the effect of IGF-I on muscle strength and power in older persons. Am J Physiol Endocrinol Metab 2003;284:E481-7.

58 Broussard SR, Zhou JH, Venters HD, et al. At the interface of environment-immune interactions: cytokine and growth-factor receptors. J Anim Sci 2001;79:E268-84.

59 Hofmann M, Halper B, Oesen S, et al. Serum concentrations of insulin-like growth factor-1, members of the TGF-beta superfamily and follistatin do not reflect different stages of dynapenia and sarcopenia in elderly women. Exp Gerontol 2015;64:35-45.

60 Haupt TH, Kallemose T, Ladelund S, et al. Risk factors associated with serum levels of the inflammatory biomarker soluble urokinase plasminogen activator receptor in a general population. Biomark Insights 2014;9:BMI.S19876-100.

61 Thunø M, Macho B, Eugen-Olsen J. suPAR: the molecular crysta ball. Dis Markers 2009;27:157-72.

62 Langkilde A, Petersen J, Henriksen JH, et al. Leptin, IL-6, and suPAR reflect distinct inflammatory changes associated with adiposity, lipodystrophy and low muscle mass in HIV-infected patients and controls. Immun Ageing 2015;12:9. 